\title{
Dose to the skin in helical tomotherapy: Results of in vivo measurements with radiochromic films
}

\author{
Michele Avanzo ${ }^{\mathrm{a}, *}$, Annalisa Drigo ${ }^{\mathrm{a}}$, Stefano Ren Kaiser ${ }^{\mathrm{b}}$, \\ Antonella Roggio ${ }^{c}$, Giovanna Sartor ${ }^{a}$, Paola Chiovati ${ }^{a}$, \\ Giovanni Franchin ${ }^{\text {, }}$, Maurizio Mascarin ${ }^{\text {, }}$, Elvira Capra ${ }^{a}$
}

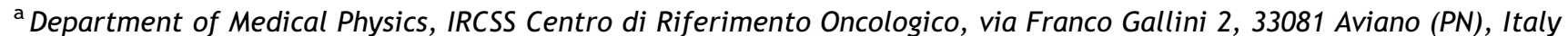

b Department of Medical Physics, Fondazione Poliambulanza Istituto Ospedaliero, Brescia 25124, Italy

' Department of Medical Physics, Veneto Institute of Oncology - IRCSS, Padua 35128, Italy

d Department of Radiation Oncology, IRCSS Centro di Riferimento Oncologico, Aviano 33081, Italy

Received 2 August 2011; received in revised form 10 April 2012; accepted 14 April 2012 Available online 8 May 2012

\section{KEYWORDS}

Intensity modulated;

IMRT;

Skin dose;

In vivo dosimetry;

Helical tomotherapy;

Radiochromic films

\begin{abstract}
Purpose: The aim of this study is to report results of measurements of dose to the skin in vivo with radiochromic EBT films in treatments with helical tomotherapy.

Methods and materials: In vivo measurements were performed by applying pieces of radiochromic films to the skin or to the inner side of thermoplastic mask before the treatment. The sites of treatment included scalp, brain, head and neck, cranio-spinal axis and lower limbs. Skin dosimetry was performed in a patient who experienced grade 3-4 acute side effects to the skin shortly after the first treatment sessions. For each patient we measured the setup errors using the daily MVCT acquired for image guidance of the treatment. EBT films were read with a flatbed Epson Expression scanner and images were processed with an inhouse written routine.

Results: A total of 96 measurements of dose to the skin performed on 14 patients. The mean difference and standard error of the mean difference between measured and TPS-calculated dose was $-9.2 \% \pm 2.6 \%$ for all treatments, $-6.6 \% \pm 2.6 \%$ for head and neck treatments. These differences were statistically significant at the 0.05 significance level ( $t$-Student test). Planned dose and dose range in the region of measurements were not correlated with dose discrepancy. Conclusions: Radiochromic EBT films are suitable detectors for surface dose measurements in tomotherapy treatments. Results show that TPS overestimates dose to the skin measured with EBT radiochromic films. In vivo skin measurements with EBT films are a useful tool for quality
\end{abstract}

\footnotetext{
* Corresponding author. Tel.: +39 (0)434 659175
}

E-mail address: mavanzo@cro.it (M. Avanzo). 
assurance of tomotherapy treatments, as the treatment planning system may not give accurate dose values at the surface.

(c) 2012 Associazione Italiana di Fisica Medica. Published by Elsevier Ltd. All rights reserved.

\section{Introduction}

Intensity modulated radiation therapy (IMRT) has improved target coverage and sparing of organs at risk compared with 3D conformal radiotherapy (3D-CRT) and is now, for many pathologies, the treatment of choice [1].

Clinical side effects to superficial structures are a major concern with radiotherapy patients, particularly in IMRT. Toxicity to the skin and mucosa can happen more frequently if chemotherapy is administered in combination with radiation treatment [2]. Clinical side effects to superficial structures in radiotherapy typically occur in 2-3 weeks after the beginning of the radiation treatment and may cause, if they are not tolerated by the patient, a treatment break [3].

Incidences of toxicities to superficial structures, such as dermatitis and alopecia have been observed with IMRT treatments [4-6]. In a study on IMRT of oropharyngeal cancer the majority of patients developed skin toxicity or acute mucosal effects of grade 3 or 4 [6]. Scalp alopecia and anterior mucositis are associated with occipital scalp dose greater than $30 \mathrm{~Gy}$ and anterior mandible dose greater than $34 \mathrm{~Gy}$ [4]. Therefore it is recommended to draw skin as a sensitive structure and reduce dose to the skin in optimization of the IMRT plan [5]. The occurrence of complications in superficial structures as skin and mucosa in IMRT is possibly related to the increased number of tangential beams compared with 3D-CRT, resulting in more non-target tissues irradiated [4]. The dose to the skin could be higher in IMRT than in 3D-CRT also because, during the inverse planning, it is required to cover the PTV with the prescribed dose even in areas where build-up effect occurs [7].

Helical tomotherapy (Hi-Art II Tomotherapy System, Tomotherapy Inc., Madison, Wisconsin, USA), is an intensity modulated radiation therapy (IMRT) modality in which a $6 \mathrm{MV}$ LINAC is mounted on a ring gantry that continuously rotates during the treatment. At the same time the couch is translated through the rotating beam plane so that the dose is delivered in a helical fashion. The beam intensity is modulated by a 64-leaf binary multileaf collimator (MLC). With this combination of MLC and table speed a highly conformal dose distribution can be achieved. The Tomotherapy system can acquire helical mega-voltage computed tomography (MVCT) images for daily image guidance using a lower energy (3.5 MV nominal) imaging beam, generated by the same LINAC that produces the therapeutic beam, coupled with an arc-shaped xenon detector array mounted on the rotating slip ring opposed to the radiation source $[8,9]$. After initial patient setup, a MVCT scan is acquired and fused with the planning computed tomography (kVCT) to determine couch adjustment, resulting in high precision positioning $[10,11]$.

By means of helical tomotherapy it is possible to create treatment plans that deliver prescribed doses even in regions close to the surface without the use of bolus [12-15]. For this reason helical tomotherapy has been used for treatments in which the skin is part of the PTV such as total scalp or whole breast irradiation $[12,16]$. Because of the high number of tangential fields in tomotherapy, dose to the skin could be higher than in fixedgantry IMRT, as confirmed by measurements of dose to the skin in vivo [17]. In a study on nasopharyngeal cancer treated with helical tomotherapy, the side effects occurring more frequently were skin and mucosal reactions [18]. Therefore, given the importance of side effects to the skin in IMRT, accurate knowledge of dose to superficial structures is critical.

The reliability of planning system dose calculations at shallow depths is also a potential issue with intensity modulated treatments involving superficial structures. An overestimation of surface dose in phantom by the TPS of up to $14 \%$ has been reported for helical tomotherapy $[13,14,16,19-22]$. In a recent work, a significantly lower dose than TPS calculations was measured on a cheese phantom using optical stimulated luminescence (OSL) dosimeter, Si diodes, MOSFETs, and radiochromic films [23]. Several studies in vivo have also demonstrated that the TPS overestimate skin dose in tomotherapy treatments [13,24,25].

Dosimeters that have been used to evaluate superficial doses in vivo and in phantom for IMRT and tomotherapy include TLDs [15,26], diodes [27], and MOSFETs [14,22,24,26]. Radiochromic EBT films (International Specialty Products, Wayne, NJ) have many attractive characteristics that make them candidates for in vivo skin dosimetry such as high spatial resolution, near-tissue equivalence, and weak energy response [28-31]. Moreover, the equivalent depth in water of the effective point of measurement for EBT radiochromic films has been determined as $153 \mu \mathrm{m}$ [29] which is very close to the clinically relevant depth for skin of $70 \mu \mathrm{m}$ recommended by ICRP [32] and ICRU [33]. In vivo measurements of dose to the skin in helical tomotherapy have been performed using TLDs $[13,15,24,25,34]$, and MOSFETS [24,25]. To our knowledge, radiochromic films have been used only for measurements of surface dose in phantom [20,21,23].

In this study we measured skin dose using radiochromic films in vivo on patients undergoing helical tomotherapy treatments with the Hi-Art TomoTherapy System treated for different pathologies, including treatments of head and neck, cranio-spinal axis (CSA), and inferior limbs. Skin dosimetry was aimed at checking delivered dose to the skin, in particular when an acute skin reaction had occurred. The aim of this study is to report the results of in vivo measurements, investigate factors possibly related to discrepancies between planned and measured dose, and study the dependence of EBT film response on the calibration method and on temperature at the time of irradiation. 


\section{Materials and methods}

\section{Film acquisition}

In film handling and storing the recommendations of the AAPM TG-55 report [35] were followed: we used gloves to avoid film soiling; minimized exposure to light and kept films together to avoid differences in thermal histories. In the present study we used films from the same batch (\# 3634802I). We scanned films with an Epson Expression 1680 Pro flatbed scanner (Seiko Epson Corporation, Nagano, Japan) [36]. The scanner was initialized by acquiring 5 blank scans and waiting 30 min warm-up time. A consistent orientation for films was maintained for all scans. Films were scanned at least $24 \mathrm{~h}$ after exposure. The central area of the scanner $\left(10 \times 15 \mathrm{~cm}^{2}\right)$ was used for film reading in order to minimize the non-uniform response of the scanner. The remaining area of the glass tray was covered by opaque radiographic films to reduce scattered light. The films were scanned in the 48-bit RGB transmission mode at 300 dpi resolution with all filter and image correction options turned off. The images were saved in a tagged image file format (TIFF).

\section{Film analysis}

The scanning of films and analysis of images were performed according to the protocol by Devic et al. [37]. Film images were analyzed with an in-house routine written with MatLab (The Mathworks, Inc, Natick, MA). For each exposed film we acquired five consecutive scans, calculated the average scan and applied a 2D Wiener filter in order to decrease image noise. The red component of each image was extracted to enhance film response. Pixels with a defective response because of the presence of specks or dust on the light pathway from the lamp to the CCD detector [37] were identified and removed from the analysis. In order to identify these pixels we acquired blank scans of the empty scanner bed taken over the same scanning region as the previously acquired images. Faulty pixels were identified as the pixels that differ by more than $5 \%$ from the maximum signal $\left(2^{16}\right)$ in the blank image. The background signal of the scanner was determined by scanning a stack of three opaque radiographic films, providing as a result a dark image. We calculated the net optical density at a given dose $D$, netOD (D), from exposed, unexposed and dark images following Devic et al. [37], as:

$\operatorname{netOD}(D)=\log _{10}\left(\frac{I_{\text {unexp }}-I_{\text {bckg }}}{I_{\exp }(D)-I_{\text {bckg }}}\right)$

where $I_{\text {unexp }}$, and $I_{\exp }(D)$, are the readings for unexposed and exposed film, while $I_{b c k g}$ is the reading from the dark image.

The uncertainty of dose was computed from the standard deviations of measured netOD and of parameters of the calibration function following the method from Devic et al. [37].

\section{Film calibration}

A calibration curve with EBT films was acquired at the TomoTherapy Hi-Art System following the procedure for calibration recommended by Tomotherapy Inc. for delivery quality assurance (DQA) of treatments. Pieces of $3 \times 3 \mathrm{~cm}^{2}$ films were exposed to a $10 \times 5 \mathrm{~cm}^{2}$ static field in a solid water phantom (Tomo Solid Water Set, Standard Imaging Inc., Middelton, WI) at $1 \mathrm{~cm}$ depth, $85 \mathrm{~cm}$ SSD. Calibration doses ranged between 20 and 560 cGy. The condition of full backscatter was guaranteed by placing a $10 \mathrm{~cm}$ thick piece of solid water under the film. Dose to the films were reconstructed from measurements performed with an A1SI Exradin thimble ion chamber placed at 1.5 depth. The average netOD was read inside a $5 \times 5 \mathrm{~mm}^{2}$ ROI centred on each exposed film. The calibration function, in terms of dose versus netOD, was fitted with the following formula [37]:

$\mathrm{D}=b \cdot \operatorname{netOD}+c \cdot \operatorname{netOD}^{n}$

where $b, c$ and $n$ are the parameters determined in the fitting process and $D$ is the dose given to the film. The asymptotic $95 \%$ confidence intervals (Cls) for the estimated parameters and $95 \%$ prediction intervals for the fitted function were evaluated, assuming the asymptotic normal distribution for the parameter estimates, from the Jacobian matrix and the residuals of fit [38].

\section{Dependence of response on the calibration method}

We acquired a calibration curve by irradiating films with a helical treatment. A tomotherapy treatment was planned on a uniform cylindrical phantom, i.e. the Cheese Phantom (Standard Imaging Inc., Middelton, WI), specifically designed for the DQA of tomotherapy treatments. The treatment plan was designed to deliver different uniform doses to the regions of interest inside the phantom. Each target volume was cylindrical-shaped and encompassed the central coronal plane of the phantom. The treatment was delivered with EBT films inserted in the central coronal plane of the phantom, and was repeated, after removal of films, with A1SI Exradin thimble ion chambers inserted into the target volumes. The doses to the films were 70,105 , $147,200,250,300,350$ and $420 \mathrm{cGy}$. The calibration curve was calculated and compared with that obtained from a static beam. We then calculated the difference in dose between the two calibration curves, and the uncertainty on dose difference by summing in quadrature the Cls on the two calibration curves.

\section{Dependence of film response on temperature}

In order to verify the change in film response with temperature at the time of irradiation pieces of radiochromic film with size $1.5 \times 1.5 \mathrm{~cm}^{2}$ were taped to the surface of a small solid water cube. The phantom was immersed in a thermostatic bath so that only its upper face, on which the radiochromic film was attached, was above the water level. The film piece was exposed to a $15 \times 15 \mathrm{~cm}^{2} 6 \mathrm{MV}$ beam produced by a Varian Clinac Trilogy (Varian, Palo Alto, CA) LINAC at an SSD of $140 \mathrm{~cm}$ and high dose rate. The treatment resulted in a total dose to the film of $3.4 \mathrm{~Gy}$ and the irradiation time was $3.3 \mathrm{~min}$. The irradiation was performed with the water and the solid phantom at room temperature $\left(22^{\circ}\right)$. 
Another EBT film was irradiated with the water temperature adjusted to keep the phantom surface at $34{ }^{\circ} \mathrm{C}$, and with a lower dose rate, so that the irradiation lasted 20 min which is the maximum duration of a Tomotherapy treatment at our institute. For each phantom's temperature 5 films were exposed and the relative netODs were calculated and converted to dose.

\section{In vivo measurements}

Skin dose verifications with radiochromic films were performed on 14 patients treated with the Hi-Art Helical TomoTherapy System. The treated volumes were scalp (2), brain (1), head and neck (7), cranio-spinal axis (CSA) (2) and inferior limbs (2). Patients were scanned with a GE Lightspeed QX/i CT scanner (GE Healthcare, Milwaukee, WI) with a $3.75 \mathrm{~mm}$ slice and $512 \times 512$ grid $(0.99 \mathrm{~mm} /$ pixel $)$ which was down-sampled to a $256 \times 256$ matrix upon import to the TPS [9] resulting in imported voxels of $1.98 \times 1.98 \times 3.75 \mathrm{~mm}^{3}$.

Five head and neck patients with metallic implants were scanned on the Tomotherapy MVCT since metallic implants cause fewer image artifacts in MVCT images compared to standard kVCT images [9]. The images set had $256 \times 256$ pixels of size $1.5 \times 1.5 \mathrm{~mm}^{2}$ and slice width $4 \mathrm{~mm}$. The treatments were planned with the tomotherapy planning software. Dose was calculated in normal grid mode which results in dose calculations for every $2 \times 2$ imported CT voxels in the axial image [9]. The prescribed dose per fraction ranged from 180 to 400 cGy. One of the patients experienced grade 3-4 radiation dermatitis (See Fig. 1). The patients were immobilized and positioned before treatment by aligning fiducials to the red movable setup lasers in the treatment room. Three external fiducials per patient were used for setup, which consisted of skin tattoos for lower limb patients, and markings on masks for scalp,

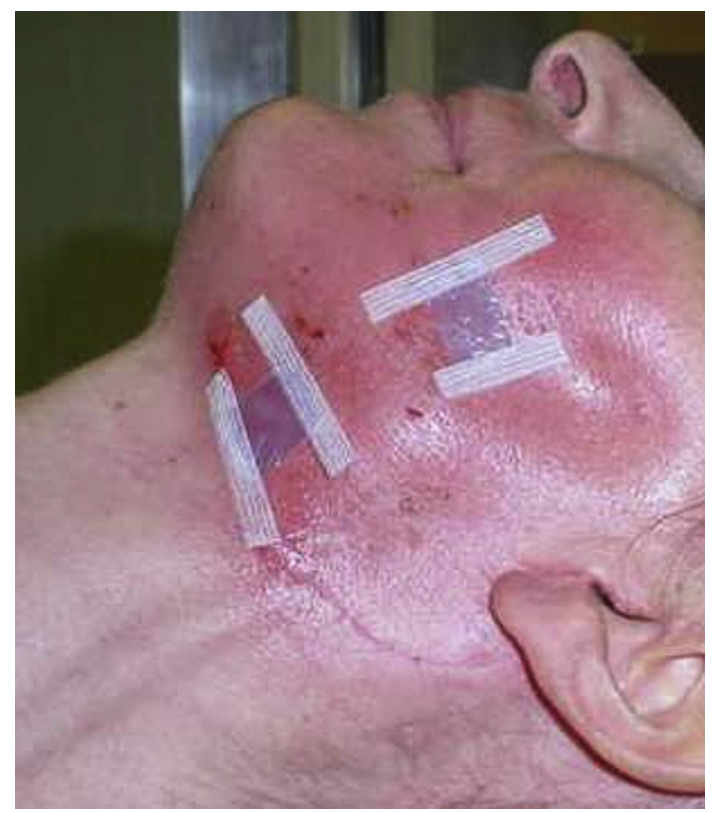

Figure 1 Skin dosimetry with radiochromic films on a patient with acute skin reaction of grade $3-4$. brain, head and neck and cranio-spinal axis patients. For all the patients in the study, MVCT images were acquired and fused with the planning image set for image guidance of the treatment. Image registration was at first performed automatically using a mutual information algorithm and then checked and adjusted manually if necessary before treatment. Positioning corrections were applied by automatic couch shifts in the longitudinal and vertical directions, manual couch shifts in the lateral direction, and roll rotations by automatic adjustment of the treatment angles.

Pieces of radiochromic film were applied to the patient's skin or to the inner side of thermoplastic mask used for immobilization. The dimensions of the film pieces varied from $1 \times 1$ to $1.5 \times 1.5 \mathrm{~cm}^{2}$. The films were applied to anatomical regions of interest for the radiation treatment; typical film positions were in correspondence to laser reference marks or anatomical markers. For some patients, the in vivo measurements were carried out over multiple treatment sessions. A total of 96 measurements were acquired. Measured doses were compared to dose to the skin on the treatment plan computed by the tomotherapy TPS. For every measurement we collected a set of values of planned doses to the skin in the voxels of the MVCT or KVCT which contained the radiochromic film. We recorded the midrange of these values and assumed it as planned skin dose. We also recorded the range of planned doses in the region of measurement. Table 1 shows, for each patient and region of interest, the planned dose, the range of planned dose values, and the number of repeated measurements over multiple fractions.

\section{Results}

The calibration curves of EBT films irradiated with a static beam and with a rotational beam are shown in Fig. 2 . The $95 \% \mathrm{Cl}$ of the dose difference between the calibration functions obtained with the two methods included zero therefore the difference was not significant. The average dose on the films irradiated at $T=22{ }^{\circ} \mathrm{C}$ was $321.8 \pm 12.0 \mathrm{cGy}$ (1SD) while for the films exposed at $T=34{ }^{\circ} \mathrm{C}$ the average dose was $316.8 \pm 13.9 \mathrm{cGy}$ (1SD) Therefore the difference was within one standard deviation of measurements.

Table 1 and Fig. 3 show the results of in vivo measurements, their location on the patients and the percentage difference from dose calculated by the TPS. When measurements were performed only in one section of a treatment, the uncertainty in absorbed dose to the film as computed from the standard deviation of measured netOD and of parameters of the calibration function [37] is shown. When measurements were repeated for more than one treatment fraction on the same patient and location, the mean \pm standard deviation of results over the repeated measurements is reported. Calculated and measured surface doses were linearly correlated (Pearson linear correlation coefficient 0.98 ).

The mean difference and standard error of the mean difference between measured and TPS-calculated dose was $-9.2 \% \pm 2.6 \%$ for all treatments. The mean absolute difference and its standard error were $10.2 \% \pm 2.4 \%$. For head and neck treatments, the mean difference was 
Table 1 Results of skin dose verifications in patients treated with helical tomotherapy. When measurements are repeated for the same patient and anatomical site during radiotherapy, the average measured dose and its standard deviation is reported. When only one measurement was performed for a patient, the uncertainty is calculated from uncertainty on measured netOD following the method from Devic et al. [37]. The last column of the table lists the percent differences between measured and calculated dose defined as (measured - calculated)/calculated dose.

\begin{tabular}{|c|c|c|c|c|c|c|c|}
\hline Patient no. & $\begin{array}{l}\text { Site of } \\
\text { treatment }\end{array}$ & $\begin{array}{l}\text { Region of } \\
\text { interest no. }\end{array}$ & $\begin{array}{l}\text { TPS dose } \\
\text { (cGy) }\end{array}$ & $\begin{array}{l}\text { TPS dose } \\
\text { range } \%\end{array}$ & $\begin{array}{l}\text { No. of } \\
\text { measurements } \\
\text { with EBT }\end{array}$ & $\begin{array}{l}\text { EBT dose } \\
\text { (cGy) }\end{array}$ & $\begin{array}{l}\text { Difference } \\
\text { EBT - TPS \% }\end{array}$ \\
\hline 1 & $\mathrm{H \& N}$ & 1 & 394 & 2.1 & 1 & $405 \pm 14$ & 2.8 \\
\hline 2 & $\mathrm{H \notin N}$ & 1 & 92 & 39.1 & 11 & $93 \pm 7$ & 1.1 \\
\hline 2 & $\mathrm{H \& N}$ & 2 & 132 & 3.1 & 11 & $129 \pm 8$ & -2.3 \\
\hline 3 & $\mathrm{H \& N}$ & 1 & 54.5 & 27.5 & 9 & $42 \pm 4$ & -22.9 \\
\hline 4 & $\mathrm{HEN}$ & 1 & 207 & 19.3 & 1 & $179 \pm 9$ & -13.5 \\
\hline 4 & $\mathrm{HEN}$ & 2 & 212 & 14.2 & 1 & $202 \pm 10$ & -4.7 \\
\hline 5 & H\&N & 1 & 164 & 26.8 & 7 & $164 \pm 8$ & 0.0 \\
\hline 6 & H\&N & 1 & 129.5 & 8.5 & 8 & $124 \pm 9$ & -4.2 \\
\hline 6 & H\&N & 2 & 127 & 10.2 & 8 & $120 \pm 11$ & -5.5 \\
\hline 7 & $\mathrm{H \& N}$ & 1 & 13.5 & 7.4 & 4 & $12 \pm 1$ & -11.1 \\
\hline 7 & $\mathrm{H \& N}$ & 2 & 153.5 & 3.3 & 1 & $134 \pm 11$ & -12.7 \\
\hline 8 & brain & 1 & 271 & 1.5 & 1 & $287 \pm 18$ & -5.9 \\
\hline 9 & scalp & 1 & 226 & 6.2 & 1 & $212 \pm 8$ & -6.2 \\
\hline 9 & scalp & 2 & 214 & 5.6 & 2 & $214 \pm 11$ & 0.0 \\
\hline 10 & scalp & 1 & 158 & 3.2 & 4 & $147 \pm 4$ & -7.0 \\
\hline 11 & CSA & 1 & 86.5 & 54.3 & 3 & $81 \pm 10$ & -6.4 \\
\hline 12 & CSA & 1 & 146.5 & 4.8 & 5 & $112 \pm 10$ & -23.5 \\
\hline 12 & CSA & 2 & 40.5 & 49.0 & 2 & $37 \pm 4$ & -8.6 \\
\hline 13 & lower limb & 1 & 183 & 5.5 & 7 & $124 \pm 7$ & -32.2 \\
\hline 14 & lower limb & 1 & 179.5 & 5.0 & 9 & $120 \pm 13$ & -33.1 \\
\hline
\end{tabular}

$-6.6 \% \pm 2.6 \%$ and the mean absolute difference was $7.4 \% \pm 2.3 \%$. All these differences were statistically significant at the 0.05 significance level ( $t$-Student test).

The difference between measured and calculated surface dose was not influenced by the presence of

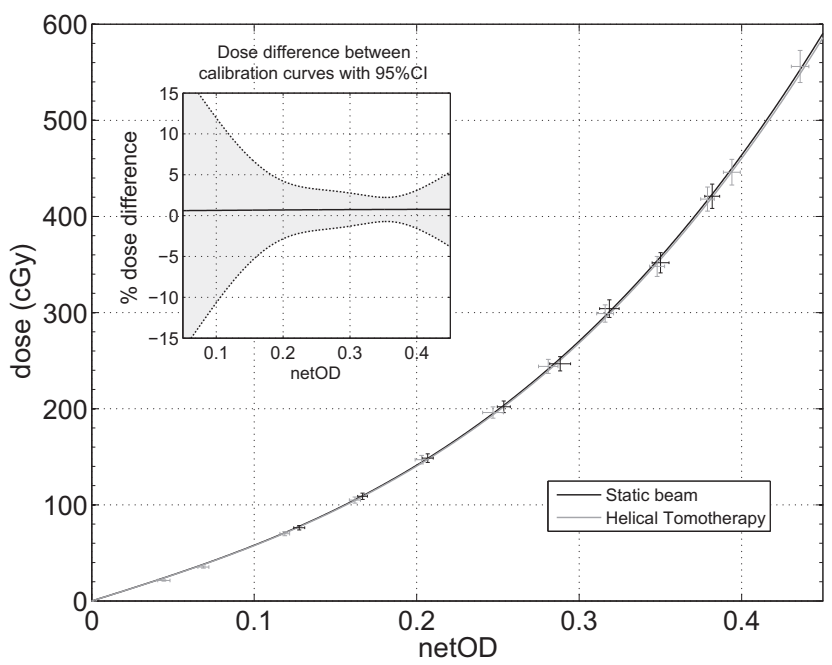

Figure 2 Calibration curves of radiochromic films acquired with static field (red) and helical tomotherapy (blue) in terms of dose versus net optical density (netOD). Error bars indicate 95\% Cls for measured values of netOD and dose. The $95 \% \mathrm{Cls}$ on calibration doses were assumed as 3.0\% [45]. Uncertainties on netOD were estimated for each scanned film following the method from Devic et al. [37]. a thermoplastic mask (Mann-Whitney $u$-test $p=0.61$ ). Two measurements in vivo were performed on a patient who experienced acute side effects to the skin (patient no. 4 in Table 1). For these measurements the differences from the calculated doses were $-13.5 \%$ and $-4.7 \%$.

\section{Discussion}

Radiochromic films are usually calibrated with a static beam perpendicular to film surface. The arrangement of beams is completely different in a typical tomotherapy

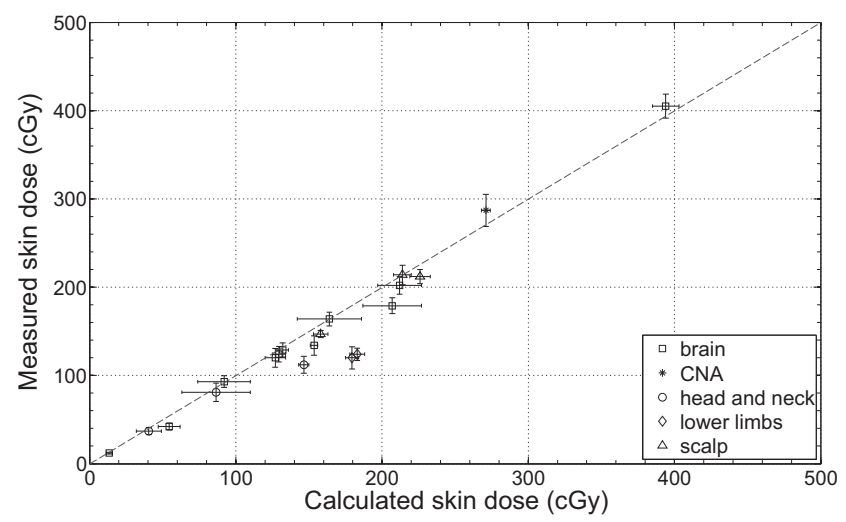

Figure 3 Skin doses measured with EBT films compared with doses calculated by the helical tomotherapy TPS, after averaging results over repeated measurements on the same patients and anatomical sites. 
treatment, where radiation is delivered over $360^{\circ}$. Some authors reported a maximum increase in response of radiochromic films of $10 \%$ when the incident beam is parallel to the film [39]. Therefore the change in film response with the calibration method was quantified before using EBT films for in vivo skin dosimetry. Given that film response was independent from the method for calibration (Fig. 2), the simpler and faster static beam calibration method can be used to determine the calibration curve.

Another concern with in vivo use of radiochromic films is dependence on temperature at the time of irradiation. Films are usually calibrated at room temperature $\left(T \approx 22^{\circ}\right)$ and, for in vivo measurements, are irradiated attached to the patient's skin $\left(T \approx 34^{\circ}\right)$ for the duration of a treatment that can last for up to $20 \mathrm{~min}$. In intra-operative radiotherapy, a correction of 0.95 to in vivo dose measured with radiochromic MD-55 films was introduced to account for temperature dependence [40]. We measured the dependence of dose reading on this change of temperature and irradiation time for EBT radiochromic films. The test revealed no significant dependence in film response. As a consequence no correction factor for temperature was applied to results of in vivo measurements.

Results of radiochromic film measurements show that the tomotherapy TPS dose algorithm overestimates superficial dose, in agreement with earlier findings in vivo and in phantom [13,14,16,19,20,22-25]. In particular, our results on head and neck patients are consistent with previous results of dosimetry of tomotherapy head and neck treatments on phantoms $[13,14,19]$ and in vivo $[13,22,24,25]$.

A reason for dose discrepancy is the difficulty of TPS to accurately calculate dose to the surface. In helical tomotherapy the dose calculation is performed using a convolution-superposition algorithm based on a collapsed cone approach [41,42]. Dose calculation inaccuracy at the air tissue interface in helical tomotherapy may originate from the assumed shape of the dose kernel near the surface in the TPS dose algorithms [43], or the inability of TPS to account for the contribution of contaminant electrons to the surface dose [44].

The helical tomotherapy TPS approximates each rotation as 51 equally spaced and discrete gantry angles. The discretization of the dynamic delivery may be a source of discrepancy of calculated to delivered dose, as in the actual delivery the dose from individual beamlets is dispersed over an arc that can span up to $7.1^{\circ}$. The dose blurring effect is more important for heavily weighted beams, because they have a longer opening time and therefore travel larger distances per gantry angle, and for beamlets that are located farther from the central axis. Therefore it could affect dose in the treatments of superficial targets, as beamlets near the surface are usually weighted more than deeper beamlets in order to achieve prescribed dose in the build-up region [43].

The limited accuracy of dose calculation at the surface could be caused by the finite size of the voxel in the patient CT scan. To each voxel at the surface is assigned one density value which is used to calculate dose, although it may contain air and tissue, two materials of extremely different density $[14,23,24]$. Spatial resolution of dose calculation is also critical [20], because with the Tomotherapy $\mathrm{Hi}$-Art beam dose increases very rapidly within the first millimeter of water [44]. In order to reduce the time needed for dose calculation and planning of treatment, in our patients the dose was calculated using a "normal" resolution grid, resulting in voxels of size $3 \times 3 \times 4 \mathrm{~mm}^{3}$ for patients scanned with MVCT, and $3.96 \times 3.96 \times 3.75 \mathrm{~mm}^{3}$ for patients planned on kVCT. These resolution grids are inadequate to accurately calculate dose in the build-up region. A "fine" calculation mode is available in the helical tomotherapy planning system, which results in a dose calculation grid that equals the imported CT data grid [9]. The usage of a fine resolution grid has been shown to improve the accuracy of calculated dose to the surface [20]. In vivo measurements with radiochromic films can also provide valuable skin dose information, as they have an effective point of measurement near to the clinically relevant depth for skin dose assessments of $70 \mu \mathrm{m}$ [29].

The highest percentage differences between measured and calculated doses were found in treatments of lower limbs ( $-32 \%$ and $-33 \%$ average). In order to find the reasons of these discrepancies, we investigated whether a change in patient anatomy occurred in the course of the radiotherapy treatment of the patient. By using the TomoTherapy Planned Adaptive software (HI-ART ${ }^{\circledR}$ version 3.1.5.3) we verified that no change in patient anatomy was observed around the point of measurement.

It has been hypothesized that in Tomotherapy treatments the discrepancy between planned and measured superficial dose is larger when more normally incident beams are used, because the dose gradient is greater in this scenario compared with obliquely incident beams [21]. In planning tomotherapy treatments of the lower limbs, directional blocking was applied to the contralateral leg in order to limit the dose to the healthy tissue. When directional block is applied to a structure the primary beamlets are not allowed to enter if the directionally blocked structure lies proximal to the target volumes. Therefore presumably inferior limb plans used more normally incident beams on the patient surface than other treatments and this could explain the high difference between measured and calculated dose.

One of the patients in this study experienced a side effect to the skin. In this case it was particularly important to check delivered dose to the skin, and measurements of skin dose in vivo were performed on the region in which the dermatitis occurred. Measured dose was lower than planned dose of $-13.5 \%$, a result that was well within the range of results obtained on other patients. Therefore we were assured that no error in treatment delivery had occurred which could cause an increase of dose to the skin. Re-optimization of the plan with stricter limits to superficial structures was then considered in order to further decrease the dose to the skin in the remaining fractions of the treatment.

\section{Conclusions}

Radiochromic EBT films are suitable detectors for surface dose measurements in tomotherapy treatments. The procedure of in vivo measurements with EBT films was relatively easy, and the time needed to setup for the in vivo measurements did not increase the overall treatment time. Results from in vivo measurements with EBT films confirm 
that Helical Tomotherapy TPS overestimates dose in the build-up region. In vivo skin measurements with EBT films are a useful tool for quality assurance of tomotherapy treatments, as they can provide a quantification of dose in areas where the treatment planning system may not be accurate. This is of particular importance when the patient's surface is part of the target or is the dose-limiting organ at risk of the radiotherapy treatment, or when toxicity to superficial structures occurs to the patient during the treatment.

\section{References}

[1] Lu JJ, Brady LW, Abitbol AA. Radiation oncology: an evidencebased approach. Berlin: Springer; 2008.

[2] Archambeau JO, Pezner R, Wasserman T. Pathophysiology of irradiated skin and breast. Int J Radiat Oncol Biol Phys 1995; 31:1171-85.

[3] Laskar S, Bahl G, Muckaden M, Pai SK, Gupta T, Banavali S, et al. Nasopharyngeal carcinoma in children: comparison of conventional and intensity-modulated radiotherapy. Int J Radiat Oncol Biol Phys 2008;72:728-36.

[4] Rosenthal DI, Chambers MS, Fuller CD, Rebueno NC, Garcia J, Kies MS, et al. Beam path toxicities to non-target structures during intensity-modulated radiation therapy for head and neck cancer. Int J Radiat Oncol Biol Phys 2008;72:747-55.

[5] Lee N, Chuang C, Quivey JM, Phillips TL, Akazawa P, Verhey LJ, et al. Skin toxicity due to intensity-modulated radiotherapy for head-and-neck carcinoma. Int J Radiat Oncol Biol Phys 2002;53:630-7.

[6] Daly ME, Le QT, Maxim PG, Loo Jr BW, Kaplan MJ, Fischbein NJ, et al. Intensity-modulated radiotherapy in the treatment of oropharyngeal cancer: clinical outcomes and patterns of failure. Int J Radiat Oncol Biol Phys; 2009.

[7] Thomas SJ, Hoole AC. The effect of optimization on surface dose in intensity modulated radiotherapy (IMRT). Phys Med Biol 2004;49:4919-28.

[8] Mackie TR, Balog J, Ruchala K, Shepard D, Aldridge S, Fitchard E, et al. Tomotherapy. Semin Radiat Oncol 1999;9: 108-17.

[9] Langen KM, Papanikolaou N, Balog J, Crilly R, Followill D, Goddu SM, et al. QA for helical tomotherapy: report of the AAPM task group 148. Med Phys 2010;37:4817-53.

[10] Boswell S, Tome W, Jeraj R, Jaradat H, Mackie TR. Automatic registration of megavoltage to kilovoltage $C T$ images in helical tomotherapy: an evaluation of the setup verification process for the special case of a rigid head phantom. Med Phys 2006; 33:4395-404.

[11] Zhou J, Uhl B, Dewit K, Young M, Taylor B, Fei DY, et al. Analysis of daily setup variation with tomotherapy megavoltage computed tomography. Med Dosim 2010;35:31-7.

[12] Orton N, Jaradat H, Welsh J, Tome W. Total scalp irradiation using helical tomotherapy. Med Dosim 2005;30:162-8.

[13] Tournel K, Verellen D, Duchateau M, Fierens $Y$, Linthout N, Reynders $T$, et al. An assessment of the use of skin flashes in helical tomotherapy using phantom and in-vivo dosimetry. Radiother Oncol 2007;84:34-9.

[14] Ramsey CR, Seibert RM, Robison B, Mitchell M. Helical tomotherapy superficial dose measurements. Med Phys 2007;34: 3286-93.

[15] Zibold F, Sterzing F, Sroka-Perez G, Schubert K, Wagenknecht K, Major G, et al. Surface dose in the treatment of breast cancer with helical tomotherapy. Strahlenther Onkol 2009;185:574-81.

[16] Reynders T, Tournel K, De Coninck P, Heymann S, Vinh-Hung V, Van Parijs $\mathrm{H}$, et al. Dosimetric assessment of static and helical
TomoTherapy in the clinical implementation of breast cancer treatments. Radiother Oncol 2009;93:71-9.

[17] Roland TF, Stathakis S, Ramer R, Papanikolaou N. Measurement and comparison of skin dose for prostate and head-andneck patients treated on various IMRT delivery systems. Appl Radiat Isot 2008;66:1844-9.

[18] Kodaira T, Tomita N, Tachibana H, Nakamura T, Nakahara R, Inokuchi $\mathrm{H}$, et al. Aichi cancer center initial experience of intensity modulated radiation therapy for nasopharyngeal cancer using helical tomotherapy. Int J Radiat Oncol Biol Phys 2009;73:1129-34.

[19] Higgins PD, Han EY, Yuan JL, Hui S, Lee CK. Evaluation of surface and superficial dose for head and neck treatments using conventional or intensity-modulated techniques. Phys Med Biol 2007;52:1135-46.

[20] Hardcastle N, Soisson E, Metcalfe P, Rosenfeld AB, Tome WA. Dosimetric verification of helical tomotherapy for total scalp irradiation. Med Phys 2008;35:5061-8.

[21] Goddu SM, Yaddanapudi S, Pechenaya OL, Chaudhari SR, Klein EE, Khullar D, et al. Dosimetric consequences of uncorrected setup errors in helical Tomotherapy treatments of breast-cancer patients. Radiother Oncol 2009;93: 64-70.

[22] Qi ZY, Deng XW, Huang SM, Zhang L, He ZC, Li XA, et al. In vivo verification of superficial dose for head and neck treatments using intensity-modulated techniques. Med Phys 2009;36: 59-70.

[23] Snir JA, Mosalaei H, Jordan K, Yartsev S. Surface dose measurement for helical tomotherapy. Med Phys 2011;38: 3104-7.

[24] Cherpak A, Studinski RC, Cygler JE. MOSFET detectors in quality assurance of tomotherapy treatments. Radiother Oncol 2008;86:242-50.

[25] Kinhikar RA, Murthy V, Goel V, Tambe CM, Dhote DS, Deshpande DD. Skin dose measurements using MOSFET and TLD for head and neck patients treated with tomotherapy. Appl Radiat Isot 2009;67:1683-5.

[26] Kinhikar RA. Surface dose for five telecobalt machines, $6 \mathrm{MV}$ photon beam from four linear accelerators and a Hi-Art Tomotherapy. Technol Cancer Res Treat 2008;7:381-4.

[27] Higgins PD, Alaei P, Gerbi BJ, Dusenbery KE. In vivo diode dosimetry for routine quality assurance in IMRT. Med Phys 2003;30:3118-23.

[28] Butson MJ, Cheung T, Yu PK. Weak energy dependence of EBT gafchromic film dose response in the $50 \mathrm{kVp}-10 \mathrm{MVp} \mathrm{X}$-ray range. Appl Radiat Isot 2006;64:60-2.

[29] Devic S, Seuntjens J, Abdel-Rahman W, Evans M, Olivares M, Podgorsak EB, et al. Accurate skin dose measurements using radiochromic film in clinical applications. Med Phys 2006;33: 1116-24.

[30] Bufacchi A, Carosi A, Adorante N, Delle Canne S, Malatesta T, Capparella $\mathrm{R}$, et al. In vivo EBT radiochromic film dosimetry of electron beam for Total Skin Electron Therapy (TSET). Phys Med 2007;23:67-72.

[31] Rink A, Vitkin IA, Jaffray DA. Energy dependence (75 kVp to $18 \mathrm{MV}$ ) of radiochromic films assessed using a real-time optical dosimeter. Med Phys 2007;34:458-63.

[32] International Commission on Radiological Protection. 1990 recommendations of the International Commission on Radiological Protection. Pergamon for the Commission; 1991.

[33] International Commission on Radiation Units and Measurements. Determination of dose equivalents resulting from external radiation sources: vol. 43, Part 2; 1985.

[34] Ito S, Parker BC, Levine R, Sanders ME, Fontenot J, Gibbons J, et al. Verification of calculated skin doses in postmastectomy helical tomotherapy. Int J Radiat Oncol Biol Phys; 2011.

[35] Niroomand-Rad A, Blackwell CR, Coursey BM, Gall KP, Galvin JM, McLaughlin WL, et al. Radiochromic film dosimetry: 
recommendations of AAPM radiation therapy Committee Task Group 55. Med Phys 1998;25:2093-115.

[36] Paelinck L, De Neve W, De Wagter C. Precautions and strategies in using a commercial flatbed scanner for radiochromic film dosimetry. Phys Med Biol 2007;52:231-42.

[37] Devic S, Seuntjens J, Sham E, Podgorsak EB, Schmidtlein CR, Kirov AS, et al. Precise radiochromic film dosimetry using a flat-bed document scanner. Med Phys 2005;32:2245-53.

[38] Seber GAF, Wild CJ. Estimation methods. In: Nonlinear regression. Hoboken, NJ: John Wiley \& Sons, Inc.; 1989. 2005, p. 21-89.

[39] Suchowerska N, Hoban P, Butson M, Davison A, Metcalfe P. Directional dependence in film dosimetry: radiographic and radiochromic film. Phys Med Biol 2001;46:1391-7.

[40] Ciocca M, Orecchia R, Garibaldi C, Rondi E, Luini A, Gatti G, et al. In vivo dosimetry using radiochromic films during intraoperative electron beam radiation therapy in early-stage breast cancer. Radiother Oncol 2003;69:285-9.
[41] Sterpin E, Salvat F, Olivera G, Vynckier S. Monte Carlo evaluation of the convolution/superposition algorithm of $\mathrm{Hi}$-Art tomotherapy in heterogeneous phantoms and clinical cases. Med Phys 2009;36:1566-75.

[42] Lu W, Olivera GH, Chen ML, Reckwerdt PJ, Mackie TR. Accurate convolution/superposition for multi-resolution dose calculation using cumulative tabulated kernels. Phys Med Biol 2005;50:655-80.

[43] Cheek D, Gibbons JP, Rosen II , Hogstrom KR. Accuracy of TomoTherapy treatments for superficial target volumes. Med Phys 2008;35:3565-73.

[44] Smith KS, Gibbons JP, Gerbi BJ, Hogstrom KR. Measurement of superficial dose from a static tomotherapy beam. Med Phys 2008;35:769-74.

[45] International Atomic Energy Agency. Absorbed dose determination in external beam radiotherapy: an international code of practice for dosimetry based on standards of absorbed dose to water. Vienna: International Atomic Energy Agency; 2000. 